\title{
Conocimiento e importancia en los estudiantes de Enfermería sobre el consentimiento informado en actos del cuidado de enfermería'
}

\author{
Gloria Bautista Espinel2
}

doi:10.11144/Javeriana.ie17-2.ciee

Cómo citar: Bautista Espinel G. Conocimiento e importancia en los estudiantes de Enfermería sobre el consentimiento informado en actos del cuidado de enfermería. Investig Enferm. Imagen Desarr. 2015;17(2): 67-84. http://dx.doi.org/10.11144/Javeriana.ie17-2.ciee

1. Artículo original de investigación producto académico del Doctorado de Bioética de la Universidad El Bosque, Bogotá, Colombia. Autorizado su envío para publicación mediante Comité Académico del día 16 de julio de 2014, acta 21. Recibido: 22 de agosto de 2014 . Aceptado: 16 diciembre de 2014. Disponible en linea: 2 de mayo de 2015.

2. Enfermera, Universidad Francisco de Paula Santander, Cúcuta, Colombia. Especialista en Práctica Pedagógica Universitaria, Universidad Francisco de Paula Santander; en Gerencia en Salud, Universidad del Norte, Colombia, y en Gestión Aplicada en Salud, Pontificia Universidad Javeriana, Colombia. Estudiante del Doctorado de Bioética, Universidad El Bosque, Colombia. Docente tiempo completo del programa de Enfermería, Universidad Francisco de Paula Santander. Correo electrónico: gloriabautista@ufps.edu.co 


\section{Resumen}

Introducción: el consentimiento informado (CI) está fundamentado en el principio bioético del respeto a la autonomía. Su utilidad en los actos de cuidado, con frecuencia, se relaciona más con aspectos administrativos e incluso jurídicos y se antepone a la visión deontológica en la que se fundamenta la relación enfermero-paciente. Es un acto inherente a las prácticas de cuidado que plantea un desafío para el desarrollo ético y humanístico de la disciplina. Objetivo: identificar el conocimiento e importancia que los estudiantes de enfermería tienen sobre el CI aplicado a los actos propios del cuidado de enfermería, mediante la teoría de los patrones del conocimiento en enfermería (PCE). Metodología: estudio descriptivo, cuantitativo trasversal, muestra de 184 estudiantes, instrumento de diseño propio; tipo escala de Likert, con 40 items, distribuidos sobre las variables: conocimiento e importancia del CI. En la validación del instrumento se realizó: validez de contenido mediante juicio de expertos, evaluación de propiedades métricas mediante alfa de Cronbach (coeficiente de 0,838) y prueba piloto con 21 estudiantes. Para el análisis de los datos se utilizó el softwate SPSS versión 22.0.0 y la información se presentó en diagramas de dispersión matricial; historial de conglomeración y dendogramas, aplicando análisis tipo clúster. Resultados: no hubo diferencias en los valores entre hombres mujeres. Las tipologías de clúster desarrolladas generaron cuatro clasificaciones taxonómicas dentro de la conceptualización del CI. Conclusión: Con el apoyo de la teoría de los PCE se determinaron los patrones del conocimiento que están más favorecidos y cuáles tienen un desarrollo incipiente. Se da poca importancia al documento escrito, y más relevancia al proceso de suministro de información en la ejecución de actos de cuidado.

Palabras clave: ética en enfermeria; consentimiento informado; atención de enfermería; conocimiento

\section{Knowledge and importance in nursing students of informed consent in acts of nursing care}

\section{Abstract}

Introduction: informed consent (IC) is based on the bioethical principle of respect for autonomy. Its usefulness in acts of care often is more related to administrative and even legal aspects and overpowers the ethical vision in which the nurse-patient relationship is based. It is inherent to care practices and poses a challenge to the ethical and humanistic development of the discipline. Objective: To identify the knowledge and importance nursing students have about the IC applied to the acts of nursing care, by applying the theory of nursing's fundamental patterns of knowing. Methodology: A descriptive, cross quantitative study with a sample of 184 students and self-designed instrument; Likert-type scale with 40 items distributed on the variables: knowledge and importance of IC. The validation of the instrument was carried out through a content validation by expert judgment, evaluation of metric properties using Cronbach's alpha (coefficient of 0.838) and pilot test with 21 students. For the data analysis we used the SPSS software (Ver. 22.0.0) and the information was presented in scatterplot matrix diagrams; history of clustering and dendrograms, using cluster analysis type. Results: There were no differences in values between men and women. The developed cluster typologies produced four taxonomical categories in the IC conceptualization. Conclusion: With the support of the theory of nursing's fundamental patterns of knowing, the most favored patterns and the ones that are poorly developed were determined. Little importance is given to the written document, and more relevance is given to the process of providing information in performing acts of care.

Keywords: nursing ethics; informed consent; nursing care; knowledge 


\section{Conhecimento e importância nos discentes de Enfermagem do Termo de Consentimento Livre e Esclarecido em atos do cuido em enfermagem}

\section{Resumo}

Introdução: O Termo de Consentimento Livre e Esclarecido (TCLE) baseia-se no princípio bioético do respeito à autonomia. Sua utilidade nos atos de cuido, com frequência, relaciona-se mais com aspectos administrativos e mesmo jurídicos e antepõe-se à visão deontológica na que o relacionamento enfermeiro-paciente é alicerçada. É um ato inerente às práticas de cuidado que coloca um desafio para o desenvolvimento ético e humanístico da disciplina. Objetivo: identificar o conhecimento e importância que os discentes de enfermagem têm sobre o TCLE aplicado aos atos relativos ao cuidado em enfermagem, mediante aplicação da teoria dos padrões de conhecimento da enfermagem (PCE). Materiais e métodos: estudo descritivo, quantitativo transversal, amostra 184 discentes, instrumento de desenho próprio; tipo escala de Likert, com 40 itens, distribuídos sobre as variáveis: conhecimento e importância do TCLE. Na validação do instrumento realizou-se: validação de conteúdo por pareceres de peritos, avaliação de propriedades métricas usando alfa de Cronbach (coeficiente de 0,838 ) e teste piloto com 21 alunos. Análise e tabulação: software SPSS apresentado em diagramas de dispersão matricial; historial de conglomeração e dendogramas, aplicando análise tipo cluster. Resultados: não houve diferença nos valores entre homens e mulheres. Os tipos de cluster gerou quatro classificações taxonômicas desenvolvidas no conceituação do TCLE. Conclusão: Com apoio da teoria dos PCE determinaram-se os padrões do conhecimento que foram mais favorecidos e quais têm desenvolvimento incipiente. Deu-se pouca importância ao documento escrito e maior relevância ao processo de fornecimento de informação no exercício de atos de cuidado.

Palavras-chave: ética em enfermagem; consentimento informado; atenção em enfermagem; conhecimento 


\section{Introducción}

El consentimiento informado (CI), aplicado a los actos propios del cuidado en enfermería, es un proceso que poco se ha trabajado de forma explícita en el marco del desarrollo disciplinar. Está integrado a los actos que el profesional desarrolla y hace parte del principio bioético, de respeto por la autonomía.

En Colombia, su aplicación en la profesión se sustenta en las leyes 266 de 1996 (1) y 911 de 2004 (2). Por otra parte, el código deontológico de la Clasificación Internacional de Enfermedades (CIE) para la profesión de enfermería, revisado en 2012 (3), reafirma la responsabilidad de enfermería en su obtención.

Su aplicación le permite al paciente con capacidad de decisión el reconocimiento del derecho, a tener criterio propio, sobre las diferentes opciones que se le deben presentar, ya sea sobre su diagnóstico, las posibilidades terapéuticas disponibles a su alcance y el papel activo que él mismo desempeña en el proceso de toma de decisión.

$\mathrm{El}$ acto de obtener el CI por parte del profesional de enfermería demanda de este una conducta ética, conocimiento del marco legal y de los momentos de la atención en que este aplica, junto a la disposición humanística, que se integra a su gestión, de tal manera que la información suministrada sea clara, precisa, completa y veraz. Pero, cabe preguntar: ¿cuál sería la información adecuada? ¿Es igual la cantidad y contenidos de información para todas las personas? ¿El CI aplicará, a todos los actos de cuidado que enfermería realiza? ¿Es necesario que en todo acto este se realice por escrito? ¿En cuáles actos se requiere formato especial? ¿Debe existir un documento exclusivo para la profesión de enfermería?

Las respuestas a estas preguntas no son claras y se requiere, en gran medida, indagar todos los aspectos que en un momento dado son necesarios para la toma de decisión por parte del paciente. Así mismo, no se puede correr el riesgo de sobrecargar al paciente con exceso de información que confunda, atemorice o genere rechazo, frente a la acción de cuidado planteada.

Las anteriores acciones implican saber, conocer y comprender el conjunto de prácticas de cuidado que involucran sus diferentes formas de implementación. En muchas oportunidades, el profesional de enfermería contribuye a la obtención del CI para otros profesionales. En otras, esta responsabilidad se le delega directamente, sin que esto implique que está trabajando este proceso en función de los actos propios de la profesión. Así, se desconoce que esta actuación no corresponde al ámbito de acción de enfermería.

El actual esquema de atención en salud colombiano ha trasformado el papel del CI, al punto que es visto más como como documento de tipo administrativo que se debe diligenciar, dado el carácter legal que este reviste. Pero no se comprende el real proceso que este involucra y se deja de lado la trascendencia que su obtención tiene para la disciplina y los futuros profesionales de enfermería.

Los estudiantes de pregrado deben conocer y establecer la importancia del CI de tal forma que su correcta aplicación les permita fortalecer los elementos éticos y bioéticos que se están implementando en los programas de enfermería. En una práctica asistencial, cada vez más despersonalizada, 
urge incorporar estos elementos para que el ejercicio profesional no se diluya únicamente en el conocimiento teórico-práctico de los procesos y procedimientos de cuidado.

El marco legal colombiano y el contexto bioético desde el cual se ha desenvuelto el CI ha generado procesos y actividades en las instituciones de salud, para que estas trabajen en la tarea de brindar a sus usuarios el trato que la ética, la bioética y el marco legal establecido por el Sistema Obligatorio de Garantía de Calidad en Salud (4) reclama.

Durante la fase de construcción de contexto local — desarrollada mediante entrevistas con las coordinadoras de enfermeria de siete instituciones prestadoras de servicios de salud (IPS) - , en el marco de la presente investigación, se evidenció la presencia de "modelos de CI informado de dos tipos: formatos de consentimiento informado exclusivos para el profesional de enfermería usado en procesos específicos como: venopunciones, aplicación de hemoderivados, postura de catéter drum y aplicación de quimioterapia”. Así mismo, se identificó en el ámbito ambulatorio la existencia de un formato de uso general, es decir, que podía ser utilizado por todos los profesionales del equipo de salud, tal es el caso del CI que se aplica en la preprueba de $\mathrm{VIH} /$ sida durante la atención prenatal (utilizado por bacteriólogos, psicólogos, médicos o enfermeros).

En las IPS consultadas no se encontró CI en enfermería como documento integrado a los anexos de las historias clínicas, tal y como lo plantea el artículo 11 de la Resolución 1995 de 1999 (5), y no se evidenció en los procesos de atención de enfermería la existencia de procedimientos documentados en relación con este. ${ }^{3}$

En enfermeria, Bárbara Carper (6) fue la primera en considerar cuatro patrones del conocimiento enfermero: empírico (o científico), personal, ético y estético, que requieren procesos diversos de educación-aprendizaje permanente e interrelacionado a fin de formar a un profesional en condiciones de responder a la complejidad de los desafíos que enfermería debe enfrentar:

El marco teórico conceptual de Carper, considerado de gran impacto ya que aborda desde una nueva perspectiva la relación arte y ciencia de enfermería a través de los "PATRONES DE CONOCIMIENTO FUNDAMENTALES EN ENFERMERÍA" (PCE). Este se sustenta en cuatro patrones: Empírico (ciencia de la enfermería), ético (componente moral), El personal o émico (identidad) y el estético (el arte de enfermería). Cada patrón de conocimiento tiene una expresión formal en palabras o teórica y la expresión en acciones o en la práctica (nondiscursove). (Adaptado de [7])

Carper consideró cuatro formas de conocer propias de la enfermería, las cuales llamó patrones de conocimiento de enfermería (PCE):

3. Fase de construcción del contexto mediante entrevista formal realizada a las coordinadoras de enfermería de siete IPS públicas y privadas de la ciudad de Cúcuta durante el desarrollo de la investigación: Conocimiento e importancia que los estudiantes de enfermería de la UFPS tienen sobre consentimiento informado aplicado en actos propios del cuidado de enfermería 2013. 
el empírico, el ético, el del conocimiento personal, y el estético. El patrón empírico tiene un carácter teórico-científico y de predicción de hechos; el patrón ético, uno de creencias y valores morales; el patrón personal; uno de autenticidad del proceso interpersonal entre enfermera(o) y paciente, y el patrón estético, uno sobre lo que es significante en cada comportamiento individual del paciente, sobre el arte en el acto de enfermería y del cuidado buscando hacer el análisis desde la propuesta de Bárbara Carper en 1978, ajustada por María D. de Villalobos 1998 sobre los patrones del conocimiento en enfermeria, tales como formas de expresión y de evidencia del tipo de conocimiento que produce enfermería; para el presente estudio se utilizaron los cuatro patrones propuestos. Adicionalmente se integraron a los procesos propuestos por Bárbara Carper en 1978, los ajustes estructurados por María D. de Villalobos 1998, relacionados con comunicación y credibilidad. (8)

Es fundamental que el profesional de enfermería, además de poseer conocimientos científicos, posea aptitudes comunicacionales y actitudes para empatizar y compartir con el paciente. Como lo plantea Lopera de Peña (9): "conocer sus características personales y necesidades de información específicas, así sabrá no solo que debe decir sino también cómo decirlo".

De esta manera será posible contribuir a la construcción ética del ejercicio del futuro profesional de enfermeria, y que esta construcción sirva de guía para el fortalecimiento de la profesión. En lo académico, estos elementos deben ser reforzados, integrados e investigados por los docentes, para así replantear los abordajes que la formación del futuro profesional en enfermería requiere.

\section{Metodologia}

La investigación realizada es de tipo descriptivo-cuantitativo de corte trasversal en la que se indagó sobre los conocimientos e importancia que los involucrados daban a las cuestiones planteadas en su objetivo, todo ello inmerso en el concepto de CI, aplicando la teoría de los patrones del conocimiento en enfermería.

La muestra correspondió a 184 estudiantes seleccionados a través de un muestreo no probabilístico por conveniencia y que cumplian con los criterios de inclusión (estudiantes del programa de enfermería durante el primer semestre de 2013, y que voluntariamente participaran).

Para el desarrollo de la presente investigación se elaboró un instrumento de recolección de datos, mediante el diseño de un cuestionario tipo escala de Likert, que constaba de 40 preguntas. El instrumento fue sometido a prueba de juicio de expertos, con los objetivos de examinar si las instrucciones y los items formulados eran comprendidos a cabalidad, de evaluar el vocabulario utilizado, de analizar la funcionalidad del orden y secuencia de las preguntas o de detectar preguntas o ítems que tuvieran escasa utilidad para los objetivos de la investigación. Se evaluaron propiedades métricas del instrumento mediante alfa de Cronbach y resultó un coeficiente $\alpha=0,838$. 
Las preguntas en el instrumento presentaron la siguiente distribución, para lo cual se tomaron en cuenta los cuatro patrones del conocimiento de enfermería, de acuerdo con los ajustes realizados por María Durán de Villalobos, en 1998 (tabla 1).

TABLA 1. Patrón de preguntas variables: conocimiento e importancia encuesta sobre consentimiento informado (UFPS 2013) desarrollada sobre la base de los patrones del conocimiento en enfermería

\begin{tabular}{|c|c|c|c|c|c|}
\hline \multirow{3}{*}{ Patrón } & \multicolumn{4}{|c|}{ Proceso } & \multirow{3}{*}{ Producto } \\
\hline & \multirow{2}{*}{$\begin{array}{l}\text { Formas de } \\
\text { conocer }\end{array}$} & \multicolumn{3}{|c|}{ Formas de hacer/comunicar } & \\
\hline & & $\begin{array}{l}\text { Simbolizar/ } \\
\text { comunicación }\end{array}$ & $\begin{array}{l}\text { Entender/ } \\
\text { credibilidad }\end{array}$ & Crear & \\
\hline Empírico & $(18-14)$ & $(20-21)$ & $(1-6)$ & $(38-26)$ & Ciencia \\
\hline Estético & $(11-15)$ & $(13-23)$ & $(24-27)$ & $(37-25)$ & Arte \\
\hline Personal & \multirow{2}{*}{$(30-16)$} & \multirow{2}{*}{$(17-19)$} & \multirow{2}{*}{$(8-9)$} & \multirow{2}{*}{$(29-39)$} & \multirow{2}{*}{ Identidad } \\
\hline Émico & & & & & \\
\hline Ético & $(3-7)$ & $(12-22)$ & $(2-4)$ & $(5-10)$ & Moral \\
\hline \multicolumn{6}{|c|}{ Preguntas que miden la variable importancia, encuesta $\mathrm{CI}$} \\
\hline Importancia & $(35-34)$ & $(31-32)$ & $(28-40)$ & $(33-36)$ & \\
\hline
\end{tabular}

Fuente: elaboración propia

Se realizó una prueba piloto con 21 estudiantes y se encontró que los items con respuestas con algún porcentaje de dificultad en cuanto a comprensión fueron: 23, 26 y 29, que corresponden a los procesos: simbolizar/ comunicación, del patrón estético; crear, del patrón empírico, y crear, del patrón émico, ubicados todos en la variable conocimientos. Los ítems de la variable importancia no presentaron ninguna dificultad. A partir de los hallazgos y de las observaciones de los estudiantes se ajustó el instrumento en el que se presentan los ítems anteriores con menor índice de comprensión y se le realizaron tres variaciones gramaticales. Los items se ajustaron a 23, 26 y 29. En este proceso, para comprobar la lectura del instrumento y su compresión, se solicitó a los expertos su valoración final.

Para la aplicación del instrumento se tuvieron en cuenta los aspectos éticos contemplados en la Resolución 8430 de 1993 (10), y aquellos que aplicaban a la metodología del estudio seleccionada. Los estudiantes que cumplian con los criterios de selección y que accedieron a participar firmaron el CI respectivo. La investigación fue presentada al comité de investigación de la universidad, el cual aprobó su desarrollo mediante acta. Para el análisis de los datos se utilizó el software SPSS versión 22.0.0. 


\section{Resultados grupo de estudio}

\section{Caracterización de la población}

El grupo de estudio estuvo conformado principalmente por estudiantes de sexo femenino (87,5\%), en el rango de 17 a 21 años (67,9\%). Los estudiantes de sexo masculino tienen una baja presencia equivalente al 12,5\%. Solo el $2,3 \%$ de los estudiantes tiene edades entre 27 y 31 años. La edad promedio se ubicó alrededor de los 20,48 años con una baja variabilidad, representada por una desviación típica de 0,15 años. El 50\% del grupo tiene edades por debajo de 20 años. Aproximadamente, el 50\% de los estudiantes cursa los 3 últimos semestres de la carrera, los estudiantes de tercer semestre solo representan el 13,6\%.

La distribución de respuestas agrupadas por patrones del conocimiento, a partir de la teoría de Carper (1978) y tomando como referente el marco epistemológico planteado por Durán Villalobos (8) sobre las cuatro formas de conocer propias de enfermería, y que se constituyen en los fundamentos ontológicos y epistemológicos de la disciplina, se presentan por patrones así:

Patrón empírico. Dimensión: "Formas de conocer". Más del 80\% manifiesta algún desacuerdo con que el CI no se aplique a todas las acciones de enfermería del área clínica y comunitaria, sean invasivas o no. Aproximadamente el $54 \%$ manifiesta estar en alguna forma de acuerdo, con que el CI solo se aplique en procedimientos invasivos. Dimensión: "Formas de hacer/comunicar”. E1 52,2\% manifestó estar completamente de acuerdo con que los protocolos de enfermería deben incluir las intervenciones y tipo de CI.

Patrón estético. Dimensión "Formas de hacer/comunicar". Aproximadamente el $73 \%$ está de acuerdo con que en enfermería exista el CI por escrito y el 60,9\% manifestó estar en desacuerdo con que su uso esté vigente y que pueda aplicarse en áreas distintas como la asistencia en las comunidades. Dimensión "Entender", el 61,4\% expresó estar en desacuerdo con que la sola firma del paciente garantice la comprensión total de la información. El 86\% expresó estar de acuerdo que al aceptar y ser consecuente con la autonomía del paciente se trabaja en condiciones de igualdad con el enfermo. Dimensión "Crear", el 52,7\% expresó estar en desacuerdo con que exista una adecuada formación en salud sobre la manera como se debe informar y gestionar el cuidado para aplicar adecuadamente el CI escrito.

Patrón émico. Dimensión "Simbolizar". El 55\% manifestó estar en desacuerdo con que el CI firmado por el paciente solo se obtenga cuando el paciente no acepte someterse a determinado procedimiento. Cerca del $80 \%$ indicó estar de acuerdo con que los cuidados de enfermería relacionados con los dominios de confort e higiene solo requieran el asentimiento, con registró en la historia clínica.

En la dimensión "Entender", cerca del $82 \%$ están de acuerdo con que el CI no aplica en casos de urgencia manifiesta (subrogado en la familia o acompañante, así como el menor de edad o paciente inconsciente e incapaz) y en enfermedades de riesgo para la salud pública. En la dimensión "Crear", el $57 \%$ de los estudiantes manifestó estar en desacuerdo con proporcionar 
toda la información cuando se suministran tratamientos a niños; en contraposición, más del $85 \%$ expresó estar de acuerdo con que, en caso de niños, se requiere su asentimiento, pero son los padres o representantes legales quienes deben dar el CI por escrito.

Patrón ético. En el análisis descriptivo de las variables que lo componen, en la dimensión "Entender" la gran mayoría (91,3\%) expresó estar de acuerdo con que el CI está fundamentado en la ética y en la bioética; además, el $50 \%$ indicó estar de acuerdo con que el CI forma parte de los derechos del paciente. En contraparte, un $40 \%$ está en desacuerdo con esto.

\section{Importancia del consentimiento informado}

El 86,5\% del grupo expresó estar de acuerdo con que el CI es garante del respeto al enfermo. Cerca del $53 \%$ de los estudiantes manifestó su desacuerdo respecto a que para enfermería sea relevante si el enfermo acepta o rechaza los cuidados de salud y tratamiento. La figura 1 muestra los valores medios de las puntuaciones de los patrones por grupos. Destaca el valor medio más elevado es el de patrón ético de conocimiento, en comparación con el resto de patrones. El patrón con menos puntuación es el patrón empírico, cuyos valores medios oscilan entre 24 y 25 puntos.

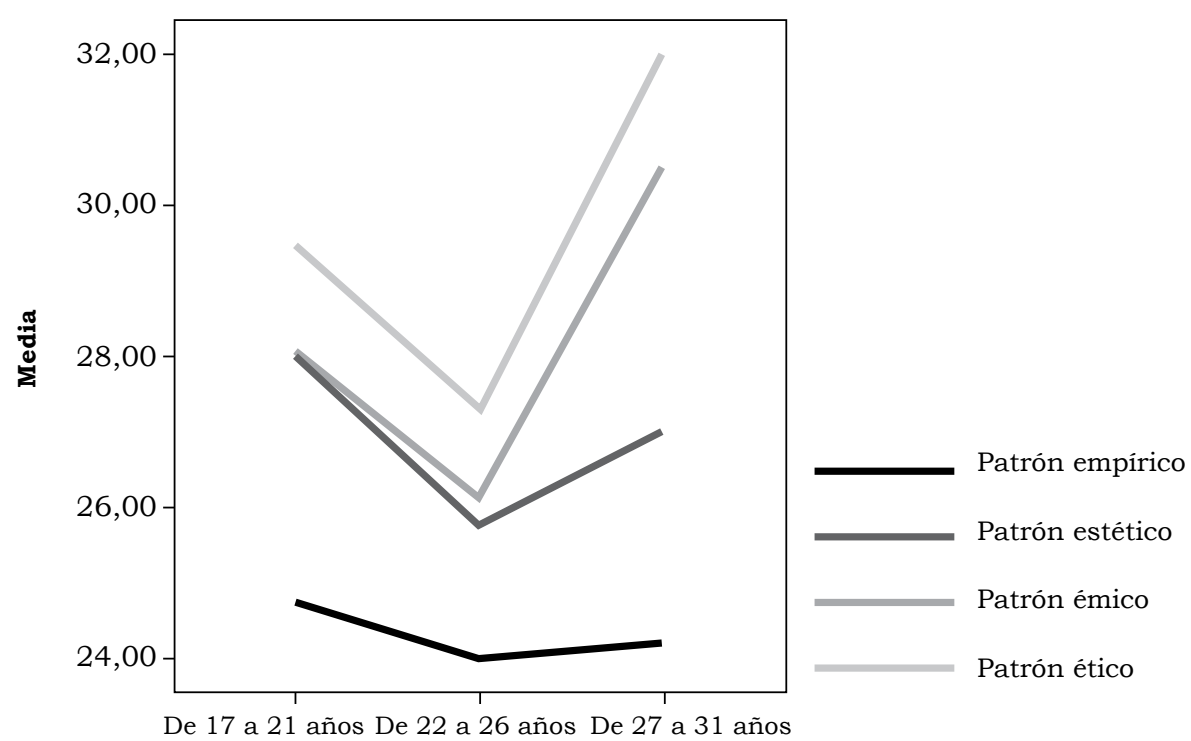

Grupos etarios

Figura 1. Puntuaciones medias en patrones de conocimiento por grupos etareos

Fuente: elaboración propia 
A la pregunta: ¿existe variación en los valores medios de cada patrón de conocimiento (empírico, émico, estético y ético) en función de los grupos? Se aplicó el análisis de varianza (Anova). Los resultados permiten plantear que existen diferencias significativas al $5 \%$ en los valores medios de las puntuaciones en los patrones estético, émico y ético. Así lo demuestran los valores de $\mathrm{p}=0,027<0,05 ; \mathrm{p}=0,022<0,05 \mathrm{y} \mathrm{p}=0,026<0,05$, que rechazan la hipótesis de igualdad de medias y permiten concluir que existen diferencias en los valores medios de los patrones de conocimientos por grupos. Un análisis post hoc de Duncan de subconjuntos homogéneos (11) permite concluir que tales diferencias son marcadas por el avance de la edad, específicamente en el grupo de estudiantes con edades comprendidas entre 27 y 31 años. Una inspección general permite apreciar un comportamiento similar al de los grupos, esto es, valores más elevados de las puntuaciones medias del patrón ético en comparación con el resto de patrones y una notable presencia de puntuaciones bajas en el patrón empírico.

Sobre la pregunta: ¿existen diferencias en las puntuaciones medias entre hombres y mujeres? Las pruebas del Anova permiten contrastar tal interrogante y muestra valores de $p>0,05(p=0,842 ; p=0,944 ; p=0,905$ y $\mathrm{p}=0,645)$, e indican que no hay diferencias significativas en los valores medios entre hombres y mujeres en lo que respecta a las puntuaciones medias de cada patrón de conocimiento. Al considerar el semestre que cursan los estudiantes (del tercero al octavo) se encuentra de forma similar que las puntuaciones medias más elevadas corresponden al patrón de conocimiento ético y que las menos ponderadas corresponden al patrón empírico. Los patrones émico y estético entrecruzan sus valores medios aproximadamente entre 26 y 29 puntos. Los resultados del Anova permiten evaluar la presencia de diferencias significativas en los valores medios de las puntuaciones de los patrones de conocimiento en función del semestre cursado. Con un valor de $\mathrm{p}=0,001<0,05$ en el patrón émico, se evidencian diferencias significativas al $5 \%$ en los valores medios de este patrón en función del semestre cursado. Un análisis post hoc de Duncan permite distinguir en qué semestre de la carrera se encuentran tales diferencias. Se puede vislumbrar que a partir del tercer semestre ya se puede distinguir una diferencia en los valores medios de las puntuaciones de los patrones del conocimiento y que se marcan principalmente en los semestres cuarto y quinto.

Con la finalidad de evaluar la intensidad de la relación para las puntuaciones de los patrones empírico, estético, émico y ético, se aplicó el coeficiente de correlación de Pearson. El resultado para las variables consideradas muestra que los patrones ético y estético se encuentran medianamente correlacionadas y que arrojan una medida de $r=0,48$. Esta interpretación también es válida entre el patrón émico y ético $(r=0,44)$. La correlación entre el patrón émico y el empírico es baja y equivale a $r=0,12$, es decir, las variaciones en una de las variables no afecta sustancialmente a la otra. La correlación entre los patrones éticos y empíricos son prácticamente nulas, pues se encuentra próxima a cero $(r=0,03)$. Se puede decir que en estos patrones se encuentran prácticamente incorrelacionadas. 
La figura 2 muestra los diagramas de dispersión para las relaciones de cada una de las variables consideradas. En ella se observa una relación lineal moderada y positiva entre los patrones émico, estético y ético; también se aprecia cómo la nube de puntos para cada par de valores considerados se aglomera en función de una línea recta. Entre el patrón empírico y el ético se observa una nube de puntos dispersa.

En un intento por identificar la estructura interna de cada patrón de conocimiento del CI, se desarrolló el análisis de clúster o análisis de conglomerados. Se adopta como método para la obtención de los grupos la "vinculación intergrupos". Los resultados se muestran de forma sistemática a través del historial de conglomeración y el dendograma. Así mismo, se presenta la interpretación de la nueva estructura y se muestran los nuevos elementos obtenidos, que encierran en sí mismos un significado teórico-práctico.

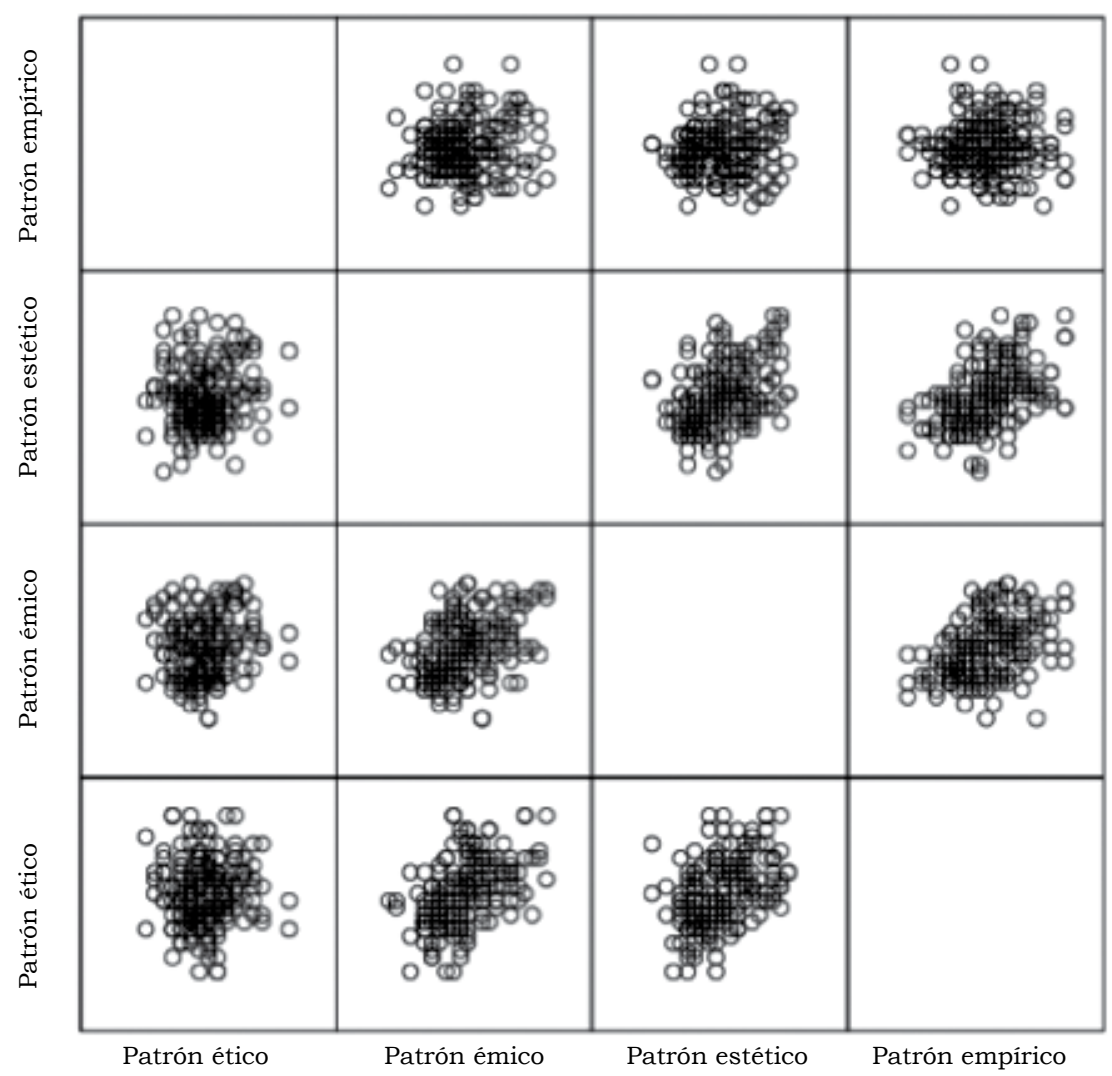

Figura 2. Dispersión matricial entre las puntuaciones de los patrones: empírico, estético, émico y ético

Fuente: elaboración propia 
Patrón estético. Para el análisis del patrón de conocimiento estético, una vez aplicada la técnica, el historial de conglomeración, en este análisis el salto más claro del coeficiente de aglomeración se observa de la etapa 3 (370) a la etapa 4 (479), lo que determina la presencia de dos grupos. En consecuencia, se considera la alternativa adecuada para representar al conjunto de variables analizadas. Al interpretar estos conglomerados, el primero corresponde a los elementos relacionados con la "operación del uso del CI"; mientras que el segundo conglomerado encierra aspectos que tienen que ver con la "gestión de la aplicación del CI". De este modo se hace una aproximación a la visión dual del patrón estético de conocimiento, que apunta hacia aspectos teóricos prácticos que hacen referencia a la operacionalización del uso y la gestión de la aplicación del CI, considerados entonces como "aspectos administrativos del CI".

Patrón émico. Del historial de conglomeración se desprende que existe un cambio importante de la etapa 3 a la 4 con un coeficiente de 375,66 a 601,00. De forma similar, la figura 3 permite percibir en la parte izquierda la clara configuración de dos clústeres que son unidos en uno solo en la etapa final. Interpretando los conglomerados obtenidos a un primer grupo se le denominó: "momentos de aplicación del CI" y el otro "condiciones y comunicación sobre el funcionamiento del CI". Ambos grupos constituyen el patrón émico, sobre el conocimiento del CI y tratan sobre los aspectos operativoscomunicacionales que abarca la aplicación del CI.

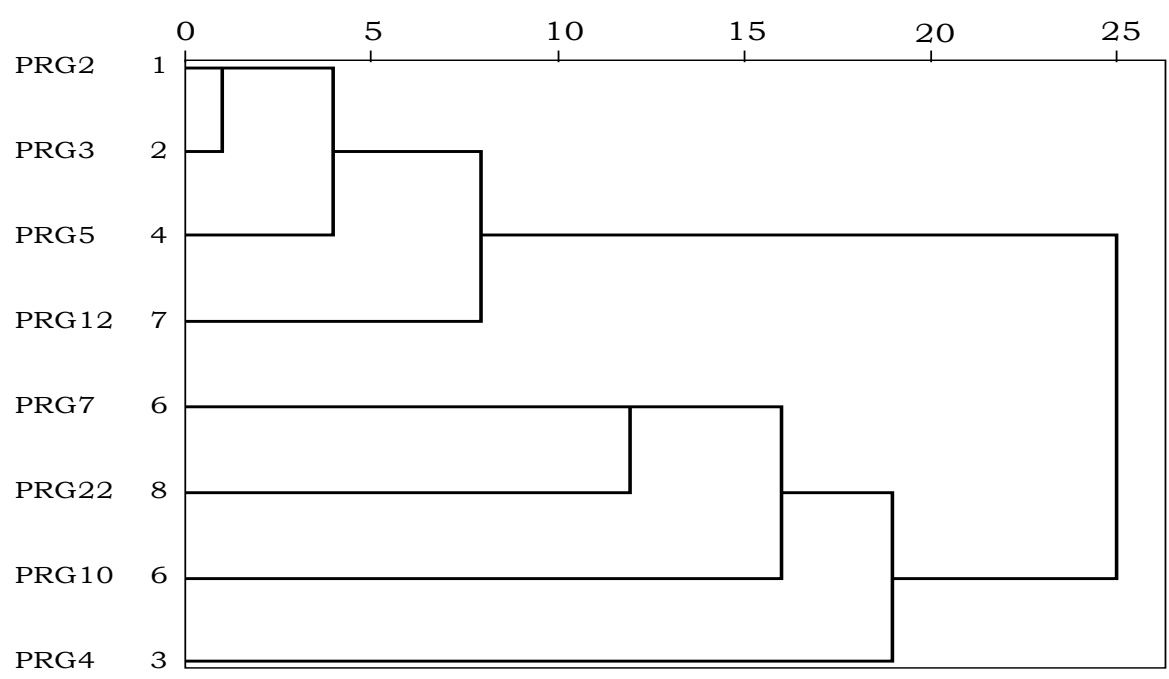

Figura 3. Dendograma para la formación de conglomerados del patrón ético de conocimiento 
Patrón ético. El historial de conglomeración para los ítems que conforman el patrón ético (figura 3) muestra su salto más significativo de la etapa 3 (384) a la etapa 4 (562) y deja clara la conformación de dos grupos; además, se aprecia en esta figura la constitución progresiva de cada uno de los grupos. El primer clúster agrupa los elementos que dan cuenta de una "visión legalista", complementada con una "referencia a los derechos humanos" en el segundo clúster se destaca una visión más humanista del CI. Ambas concepciones constituyen un marco para delimitar los derechos de los pacientes y aspectos jurídicos-legales que afectan la aplicación del CI.

Patrón empírico. Se aprecia que el salto más amplio se da de la etapa 3 (350) a la etapa 4 (466) y que marca la configuración de dos grupos. Al interpretar la configuración de los clústeres para el patrón empírico de conocimiento emerge un primer grupo denominado "conocimiento teórico del CI" y el segundo grupo se relaciona con los "procedimientos de aplicación del CI". Ambas dimensiones pueden leerse como los fundamentos procedimentales en la aplicación del CI. Para finalizar se recurrió a la técnica denominada matriz de triangulación; para ello la matriz resultante sintetizó el uso de las tres diferentes técnicas de análisis empleadas en el presente estudio: análisis descriptivo (AD), análisis factorial (AF) y análisis de conglomerados (AC). Desde esta perspectiva, y de acuerdo con lo planteado por Arias (12).

\section{Discusión}

La importancia otorgada por los estudiantes de enfermería al CI escrito es poca, ya que cerca de la mitad del grupo no ha usado nunca un formato de CI exclusivo para actos de cuidado en enfermería. Un alto porcentaje planteó su desacuerdo en el uso del CI en procedimientos invasivos de enfermería.

Similar situación se observó en los resultados obtenidos por Martín y colaboradores (13) en un estudio descriptivo realizado en la Comunidad Autónoma de la Región de Murcia. Se analizó el acto del CI por enfermeros(as) de urgencias y emergencias, y en los resultados se obtuvo que:

[...] los enfermeros/as no tienen registros documentados de consentimiento informado para procedimientos invasivos en urgencias. Se destaca que la realización de procedimientos invasivos es llevada a cabo por los Enfermeros/as sin supervisión de otro profesional en un $83 \%$ de los casos, desconociendo en un $69 \%$ de los casos la legislación vigente en materia de información al paciente. Un $43 \%$ de los enfermeros/as encuestado nunca solicita el consentimiento informado ante un procedimiento invasivo, aun asi el $93 \%$ refiere no haber tenido problemas nunca con el consentimiento informado. El resto lo solicita de forma exclusivamente verbal y como requisito ético. (13)

En la variable conocimiento por grupos de edades se evidenciaron diferencias claras en las respuestas para los patrones émico, estético y ético; pero no se observan variaciones en el patrón empírico. Existe una brecha significativa en lo abstracto del conocimiento de enfermería (teorías y modelos) que representa el patrón empírico, el cual en todos los grupos de 
edades mantienen un mismo nivel de percepción y frente a su aplicabilidad en el campo práctico.

Resultados concordantes se encontraron en el estudio de Capretti y colaboradores (14), respecto al CI: el $88 \%$ de los encuestados refiere conocer el concepto. Al interrogarlos respecto a si creen que deben realizar el CI relacionado con el riesgo del tratamiento, el $27 \%$ contestó: "ante tratamientos de elevado riesgo", el 14\% en "riesgo moderado" y el 59\% ante "cualquier tratamiento". Respecto a la decisión en el tratamiento, los encuestados consideran: "el paciente debe ser siempre informado", en el 95\% de los casos, y el $5 \%$, "solo en situaciones de riesgo". Ningún encuestado de este grupo considera que debe ser el profesional quien decide si informa o no al paciente. Con respecto a ofrecerle al paciente varias opciones para que él decida, consideran realizarlo "siempre" el 27\%, "frecuentemente" y "a veces" el $23 \%$ y en ningún caso la respuesta fue "nunca" (14).

En los patrones de respuestas existen cambios significativos, lo cual implica que, desde la perspectiva teórica de los patrones del conocimiento, hay grandes variaciones entre los estudiantes, por grupos de edad. Se encuentran principalmente variabilidades en el conocimiento ético o conocimiento moral, lo que se apreció con marcadas diferencias al momento de valorar y clarificar situaciones éticas, diferenciándolas de conceptos teóricos, que para ellos eran más claros. Esto parece ser un proceso perceptivo propio de los estudiantes, ya que en la encuesta sobre valores profesionales de los enfermeros, según la percepción de los estudiantes de enfermería en tres universidades de Bogotá.

López (15) encontró que las preguntas relacionadas con los valores éticos, morales y estéticos evidencian mayoritariamente que estos consideran extremadamente importante: proteger los derechos morales y legales de los pacientes, salvaguardar el derecho de los pacientes a la intimidad y garantizar la confidencialidad del paciente. Mientras que en el hacer frente a los profesionales con prácticas inapropiadas o cuestionables, el 17\% respuestas considera estas poco importante o nada importante, siendo este el item que con mayor frecuencia identificaron los estudiantes, como el menos importante para sus profesores. Desde su perspectiva, seguido del relacionado con la defensa del derecho de los pacientes.

El patrón émico presentó variaciones significativas entre la traducción de las valoraciones éticas y su ejecución en las representaciones prácticas planteadas. Esto está muy relacionado con la forma como el estudiante transforma el conocimiento base. Están implicados los procesos de introspección personal, dados por el ser mismo; su interiorización refleja su propio quehacer en la valoración que les dieron a las situaciones planteadas.

En el estudio adelantado por Urrutia y Alvarado (16) se hallaron similitudes en este aspecto relacionadas con los conocimientos teóricos sobre los principios elementales de bioética y algunas formas de comportamiento relacionadas con estos principios en el personal de enfermería. Es necesario ampliar la enseñanza de la ética en los estudiantes de enfermería. Es un imperativo que obedece a los avances del conocimiento y a las presiones que surgen de una cultura en las cuales el dolor, la limitación del esfuerzo 
terapéutico, la muerte y el concepto de calidad de vida, que han adquirido nuevas y perturbadoras connotaciones. Por lo tanto, es necesario formar profesionales que respondan a estas definiciones y respuestas concretas para estas nuevas problemáticas.

Para el patrón del conocimiento estético o arte de enfermería, las variaciones fueron altas y reflejan aspectos que guardan relación con la función del diseño del cuidado (crear el cuidado adaptándolo de forma individual según la valoración que este realiza), lo cual es posible, partiendo del bagaje cultural, que el estudiante le aporte y que logrará su máxima expresión a lo largo de su ejercicio profesional, dándole a este su sello individual y diferenciador.

Los anteriores resultados son opuestos a los obtenidos por Gómez y colaboradores (17), quienes describen las características de los comportamientos de cuidado identificados por estudiantes al responder un cuestionario tipo escala Likert, al que se le aplicaron pruebas de validez facial y de contenido. Se realizó un análisis teórico de los hallazgos en su relación con la epistemología de enfermería y los patrones de conocimiento de enfermeria propuestos por Carper. Los resultados señalan que "la percepción de cuidado en estudiantes de primer semestre de la Facultad de Enfermería de la Universidad Nacional de Colombia se enfoca en los comportamientos de cuidado relacionados con el patrón de conocimiento empírico", es decir, con los fundamentos de asistencia clínica y técnicas para la atención de enfermería y lo propio del patrón ético. Los patrones émico y estético se percibieron con menor frecuencia, por lo que se destaca la importancia de formar a los estudiantes en las dimensiones humanísticas del cuidado (17).

Las tipologías de clúster, de acuerdo con los coeficientes de aglomeración desarrollados, generaron la siguiente clasificación taxonómica dentro de la conceptualización del CI: para el patrón empírico las dimensiones pueden interpretarse como los fundamentos procedimentales en la aplicación del CI. El patrón estético hace referencia a la operacionalización del uso y la gestión de la aplicación del CI, considerados los aspectos administrativos del CI. El patrón ético se constituye en un marco para delimitar los derechos de los pacientes y aspectos jurídicos-legales que afectan la aplicación del CI. El patrón émico trata sobre los aspectos operativos-comunicacionales que abarca la aplicación del CI.

Sobre el particular, el Colegio de Enfermeros de Perú publicó en 2008 (18) un documento que resume la situación actual en el colectivo de enfermería en relación con el derecho a la información y el CI. Dentro de las recomendaciones más importantes están: "en el proceso del cuidado enfermero debe hacer efectivo el derecho del usuario a ser informado sobre todo procedimiento de Enfermería invasivo o no invasivo que se realizará en su cuerpo antes de proceder a efectuarlo", "Registrar en la historia clinica la información brindada y la aceptación o no de la persona", "Tener en consideración la posibilidad de no aceptación del usuario, si esto ocurriera lo recomendable es dialogar, averiguar los motivos y aclarar dudas al respecto. Si persiste la negativa, aceptarla y dejar abierta la posibilidad de que la persona pueda cambiar de parecer. Registrar en la historia clínica el hecho y las medidas tomadas", "El Consentimiento Informado no es indispensable en casos de 
emergencia, ya que es prioridad salvar la vida de la persona, por lo que la enfermera(o) actuará con el equipo de salud para adoptar las medidas necesarias y realizar las coordinaciones pertinentes".

\section{Conclusión}

Se logró con el apoyo de la teoría de los patrones del conocimiento en enfermería determinar cuáles están más favorecidos y establecer cuáles tienen un desarrollo incipiente dentro del grupo de estudio.

Respecto a la importancia que los estudiantes de enfermería le dan al CI, se evidenció que cerca de la mitad del grupo le da poca importancia al documento. Se rescata que informan al sujeto de cuidado y su familia; lo anterior induce a considerar que en pregrado es poco el uso que se ha dado al documento CI y que se privilegia el suministro de información relevante para el paciente, en la ejecución de actos de cuidado de la profesión.

\section{Conflicto de interés}

Ninguno

\section{Financiación}

Universidad Francisco de Paula Santander

\section{Referencias}

1. Ley 266/1996, por la cual se reglamenta la profesión de enfermería en Colombia y se dictan otras disposiciones. Diario Oficial 42.710, del 5 de febrero de 1996.

2. Ley 911/2012, por la cual se dictan disposiciones en materia de responsabilidad deontológica para el ejercicio de la profesión de enfermería en Colombia; se establece el régimen disciplinario correspondiente y se dictan otras disposiciones. Diario Oficial 45.693, de 6 de octubre de 2004.

3. Consejo Internacional de Enfermeras (CIE). Código deontológico para la profesión de enfermería [internet]. [Citado 2013 ago 30]. Disponible en: http://www.icn.ch/images/stories/documents/about/icncode_spanish.pdf.

4. Decreto 1011/2006, por el cual se establece el Sistema Obligatorio de Garantía de Calidad de la Atención de Salud del Sistema General de Seguridad Social en Salud. Diario Oficial. Disponible en http://www. minsalud.gov.co/Normatividad/DECRETO\%201011\%20DE\%202006.pdf

5. Resolución 1995/1999, por la cual se establecen normas para el manejo de la historia clínica. Diario Oficial 43655, agosto 5 de 1999.

6. Carper B. Fundamental patterns of knowing in nursing. Adv Nurs Sci. 1978;1(1):13-23.

7. Caballero E, Becerra R, Hullin C. Proceso de enfermería e informática para la gestión del cuidado. Madrid: Mediterráneo; 2010.

8. Durán de Villalobos M. Marco epistemológico de la enfermería. Aquichán. 2002;2(2):7-18. 
9. Lopera Peña A. Ética y bioética. Bogotá: Orión; 2005.

10. Resolución 8430/1993, por la cual se establecen las normas científicas, técnicas y administrativas para la investigación en salud.

11. Pardo A, Ruiz M. Análisis de datos con SPSS 13. Madrid: McGrawHill; 2005.

12. Arias M. Triangulación metodológica: sus principios, alcances y limitaciones. Investigación y Educación en Enfermeria. 2000;18(1):37-57.

13. Martín M, Ruiz Lavela M, Martín Rodríguez M. El consentimiento informado en enfermería de urgencias y emergencias, una visión real. Ciber Revista SEEUE [internet]. 2009;(8). Disponible en: http://www. enfermeriadeurgencias.com/ciber/julio2009/pagina6.html

14. Capretti M, Cavallo G, Michel J, Miranda M, Montaño C, Pozzo Y, Salomón S. La nueva cultura del cuidado en bioética y los estudiantes de enfermería [internet]. Documento procedente del III Foro Provincial de Investigación para la Salud-Ministerio de Salud; 2009 [citado 2013 sep 2]. Disponible en: http://www.fcm.uncu.edu.ar/jornadas2012/index.php/.

15. López A. Valores profesionales de los enfermeros profesores según la percepción de los estudiantes de últimos semestres de la carrera de enfermería en tres universidades de Bogotá [internet]. 2012 [citado 2013 sep 10]. Disponible en: http://www.bdigital.unal.edu.co/8906/1/ Arabelyl\%C3\%B3pezpereira.2012.pdf.

16. Brevis I, Sanhueza O. La bioética en la enseñanza y la investigación en enfermería. Rev Cubana Enfermer [internet]. 2007 [citado 2013 sept 15];23(3). Disponible en: http://www.bvs.sld.cu/revistas/enf/ vol23_3_07/enf07307.html.

17. Gómez O, Daza LA, Zambrano V. Percepción de cuidado en estudiantes de enfermería: caracterización e impacto para la formación y la visión del ejercicio profesional. Av Enferm [internet]. 2008;26(1). Disponible en: http://www.scielo.org.co/scielo.php?script=sci_arttext\&pid $=$ S0121-45002008000100009

18. Colegio de Enfermeras(os) del Perú. El derecho a la información, el consentimiento informado y la práctica de enfermería. Lima: Colegio; 2006-2008. 
